\title{
Ectopic prolactin-secreting adenoma at the pituitary stalk
}

\section{Katharina Schilbach \& Jochen Schopohl}

\section{Medizinische Klinik und Poliklinik IV, Klinikum der Universität München, Ziemssenstr. 1, 80336 München, Germany}

\section{Introduction}

Prolactin-secreting pituitary adenomas are the most common hormone-secreting pituitary tumors. Around 90 percent of the tumors are benign intrasellar microadenomas. The remaining cases are benign macroadenomas and in rare cases malignant prolactinomas. Very rarely benign prolactinomas are located outside the sella; in most of these cases the tumors are located in the sphenoidal sinus.

\section{Medical history}

Here we report a 35-year-old woman who presented with secondary amenorrhoea and galactorrhoea. Initial laboratory testing revealed hyperprolactinemia between 1100 and $1600 \mu \mathrm{U} / \mathrm{ml}$ and hypogonadotropic hypogonadism without further anterior or posterior pituitary insufficiency. MRI showed normal intrasellar structures, but a mass $(5 \times 6 \times 5 \mathrm{~mm})$ at the pituitary stalk just below the optic chiasm. Further investigations did not show any evidence of underlying systemic disorder or malignancy (for test results see Table $1 \&$ ).

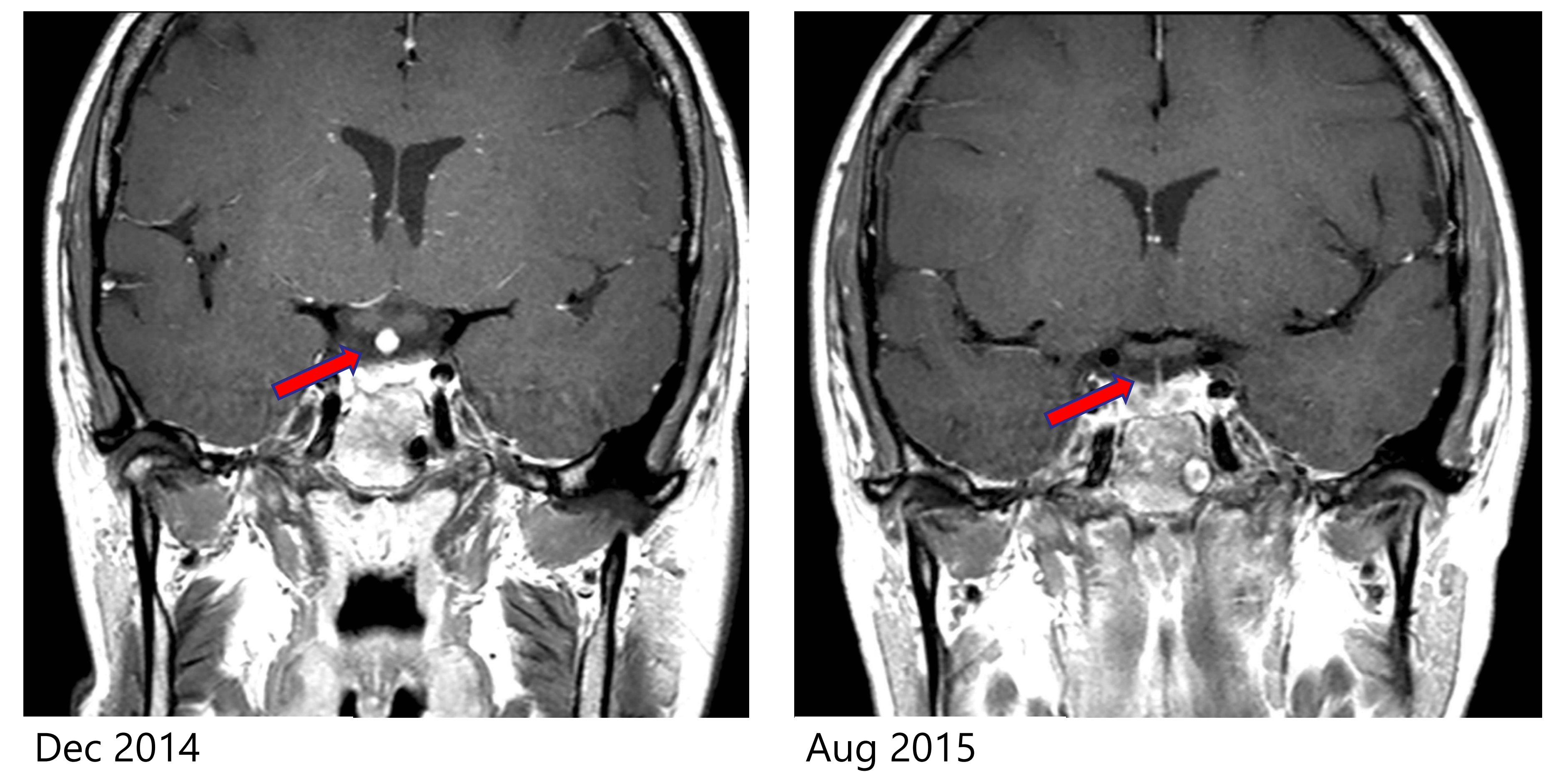

MRI scan: December 2014: a contrast-enhanced spherical mass is visible at the pituitary stalk (5 x 6 × 5 mm). August 2015: normal pituitary stalk.

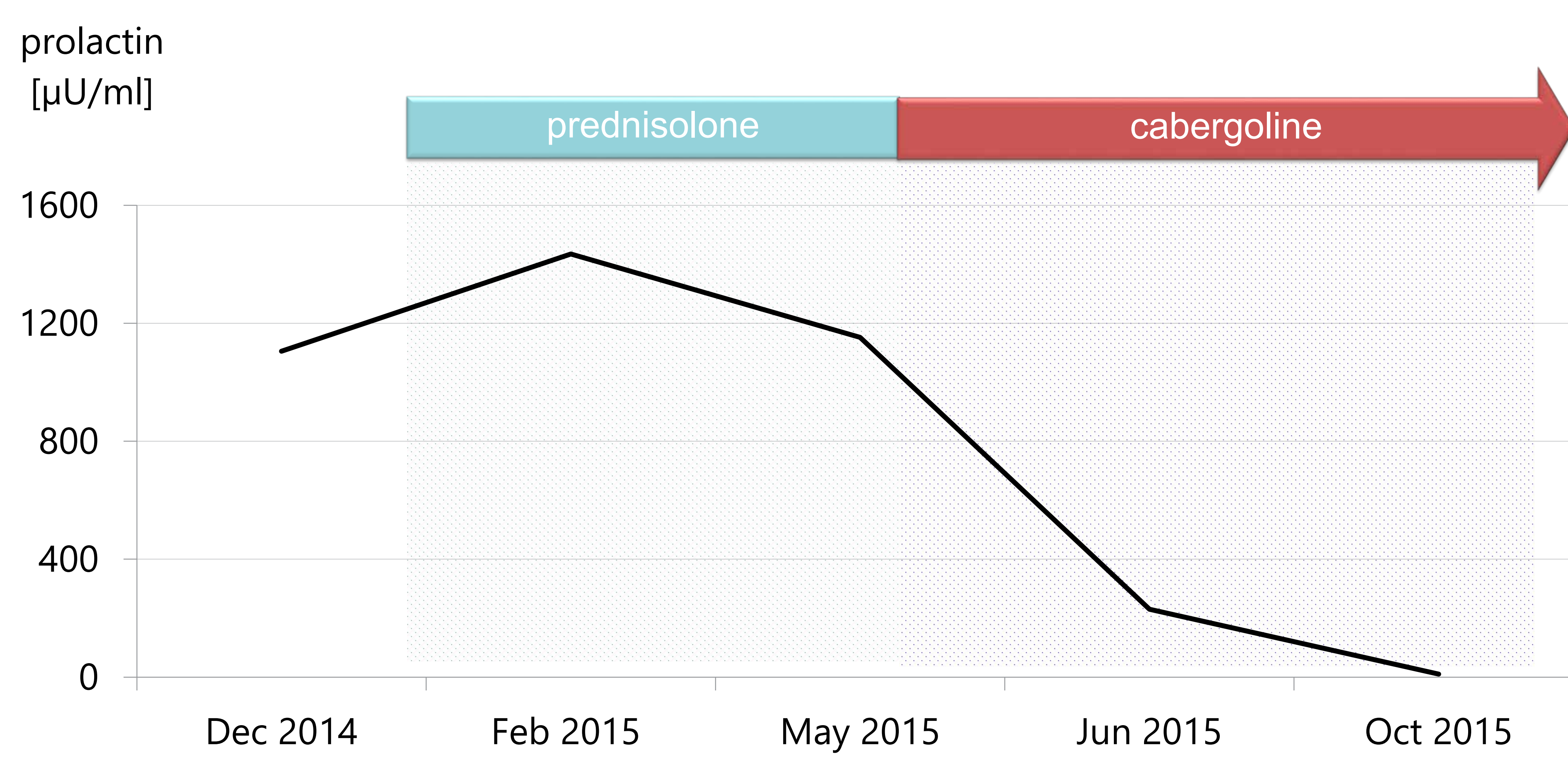

Figure 1: prolactin level

\section{Therapy}

Stereotactic biopsy was discussed, but not performed, due to the risk of severe side effects. Considering the most likely differential diagnosis of an inflammatory process such as hypophysitis or sarcoidosis (hyperprolactinemia due to compression of the pituitary stalk), the patient was treated with glucocorticoids for 5 months, which did not have any effect on the stalk mass or the laboratory findings. Treatment was then switched to low-dose cabergoline and 8 weeks later prolactin levels were normalized (see Figure 1); the tumor was no longer visible on the follow-up MRI scan and the patient had a regular menstrual cycle again. Those findings were still present in a recent check-up (May 2016).

\section{Conclusion}

Due to the course of the disease under the different pharmacological treatments, we diagnosed an ectopic microprolactinoma of the pituitary stalk, a rare entity which has been described twice so far. This case suggests that in patients with stalk lesions of unknown aetiology and hyperprolactinemia, dopamine-agonistic therapy should be considered before initiating invasive diagnostic procedures.

\section{3-EP}

